\title{
Combined characterization of a pituitary adenoma and a subcutaneous lipoma in a MEN1 patient with a whole gene deletion
}

\author{
Daniela Rusconi ${ }^{a}$, Emanuele Valtorta ${ }^{a}$, Ornella Rodeschini ${ }^{a}$, Daniela Giardino ${ }^{a}$, \\ lughetti Lorenzo ${ }^{\mathrm{b}}$, Barbara Predieri ${ }^{\mathrm{b}}$, Marco Losa ${ }^{\mathrm{c}}$, Lidia Larizza ${ }^{\mathrm{a}, \mathrm{d}}$, \\ Palma Finelli ${ }^{\mathrm{a}, \mathrm{e}, \mathrm{k}}$ \\ a Laboratory of Medical Cytogenetics and Molecular Genetics, IRCCS Istituto Auxologico Italiano, Milan, Italy; ${ }^{\mathrm{b}}$ Department of \\ Pediatrics, Università di Modena e Reggio Emilia, Modena, Italy; ${ }^{\mathrm{C}}$ Department of Neurosurgery, IRCCS Ospedale San \\ Raffaele, Milan, Italy; ${ }^{d}$ Division of Medical Genetics, San Paolo School of Medicine, Università degli Studi di Milano, Milan, \\ Italy; ${ }^{\mathrm{e}}$ Department of Biology and Genetics for Medical Sciences, Università degli Studi di Milano, Milan, Italy
}

\begin{abstract}
Multiple endocrine neoplasia type 1 (MEN1) is an autosomal dominant hereditary disorder associated with mutations of the MEN1 gene, which is characterized by combined tumors of the parathyroid glands, pancreatic islet cells, and the anterior pituitary. A significant number of patients with the clinical features of MEN1, however, do not show MEN1 mutations upon direct sequencing. We describe a young woman who fulfilled the clinical and biochemical criteria for MEN1 syndrome, but DNA sequencing did not indicate any MEN1 mutations. She developed a prolactin-secreting pituitary macroadenoma, primary hyperparathyroidism with parathyroid hyperplasia, pancreatic lesions, and two subcutaneous lipomas. Array comparative genomic hybridization $(\mathrm{aCGH})$ analysis of peripheral blood DNA revealed a heterozygous germline deletion at 11q13.1 that spanned at least 22.23 kilobases and contained the entire MEN1 gene. Integrated aCGH and cytogenetic analyses of the adenoma and lipoma tissues revealed somatic inactivation of the wild-type MEN1 allele by different routes: the second hit of MEN1 recessive oncogenesis leading to adenoma implied a loss of heterozygosity, whereas a balanced translocation deleting the wild-type MEN1 allele primed the lipoma development. These findings show that aCGH is a valuable means of optimizing genetic testing in MEN1 patients which complements other technologic approaches to elucidating the pathologic mechanisms of MEN1 tumors.
\end{abstract}

Keywords FISH, aCGH, MEN1, pituitary adenoma, lipoma

(C) 2011 Elsevier Inc. All rights reserved.

Multiple endocrine neoplasia type 1 (MEN1) is a rare autosomal dominant monogenic disorder with $95 \%$ penetrance that occurs in approximately $1 / 30,000$ people and has an equal gender distribution (1-3). It is characterized by the development of parathyroid adenomas (90-97\%), pituitary adenomas (15-50\%), and duodenal and/or pancreatic neuroendocrine tumors (30-80\%) $(4,5)$. In addition to these major lesions, patients may develop adrenal or thyroid adenomas, bronchial or thymic carcinoid tumors, and (albeit less frequently) various non-endocrine tumors such as

Received September 20, 2010; received in revised form March 25, 2011; accepted March 31, 2011.

* Corresponding author.

E-mail address: finelli@auxologico.it lipomas, angiofibromas, collagenomas, and leiomyomas $(6,7)$. The clinical diagnosis is based on the presence of at least two of the three main MEN1-related tumors $(8,9)$, and there are reports of both sporadic (de novo) and familial forms. Familial MEN1 is diagnosed in the setting of a MEN1 case and a first-degree relative with at least one of the main MEN1-related tumors; sporadic MEN1 when there is no family history of MEN1-related tumors (10).

The MEN1 causative gene (11) has 10 exons and encodes a 610 amino acid protein known as menin, a ubiquitously expressed nuclear protein that interacts with a number of the proteins $(12,13)$ involved in transcriptional regulation, genome stability, and cell division and proliferation $(14,15)$. MEN1 acts as a tumor suppressor in accordance with the "two-hit" model of hereditary cancer postulated by Knudson: 
the first hit is germline (inherited or developed during gametogenesis) and generally remains silent until the first tumor develops; the second hit leads to clonal expansion as a result of the somatic loss of the MEN1 wild-type allele in the cell target of neoplastic transformation $(9,16)$.

More than 450 MEN1 germline mutations have been identified so far (http://www.hgmd.org) (14,17), mainly spread throughout the coding region. Most of them (>80\%) are inactivating nonsense and frameshift mutations that lead to the premature truncation of menin, whereas whole or partial gene germline deletions are quite rare (1\%) (14). Nine of the reported deletions are intragenic, although a few encompass several exons and have been detected by various molecular genetic techniques [Southern blotting, reverse-transcriptase polymerase chain reaction, gene dose assays, and long-range polymerase chain reaction; reviewed in (18)]. Only two patients have been found to be constitutive carriers of a larger deletion involving neighboring genes by means of a gene dosage assay and multiplex ligationdependent probe amplification (MLPA) $(19,20)$.

MEN1 germline mutations are detected in about $68 \%$ of cases with a clinical diagnosis of familial MEN1, as well as in $26 \%$ of sporadic cases $(21-23)$. The reasons for this low detection rate are the following: 1) gross deletions that are not picked up by direct DNA sequencing; 2) the presence of mutations in the non-coding regions of the gene, which are not routinely examined; 3 ) false-negative findings due to preferential allele amplification; and 4) locus heterogeneity.

We herein describe a female patient with the MEN1 syndrome who was negative for MEN1 mutations upon DNA sequencing, although array comparative genomic hybridization $(\mathrm{aCGH})$ analysis showed that she carries an 11q13.1 germline deletion encompassing the entire MEN1 gene. She first came to clinical attention because of a prolactinsecreting pituitary macroadenoma and primary hyperparathyroidism with parathyroid hyperplasia but, during the follow-up, was found to have developed two subcutaneous lipomas and pancreatic lesions compatible with multiple localizations of a pancreatic neuroendocrine tumor.

Characterizing cryptic mutations in apparently mutationnegative patients is important because of its potential diagnostic value and the fact that it may help to improve patient management. Our findings indicate that aCGH is a useful means of identifying deletions that may be missed by conventional mutation analysis and can thus contribute toward optimizing the MEN1 mutation detection rate. Furthermore, integrating cytogenetic, FISH, and aCGH studies of lipoma and adenoma tissues can reveal the multiple routes underlying the "second hit" of MEN1 oncogenesis.

\section{Materials and methods}

\section{Clinical report}

The proband is a 23-year-old woman born of unrelated parents with no family history of endocrinopathy. Written informed consent for the research investigation, which was approved by the Ethical Clinical Research Committee of Istituto Auxologico Italiano, was obtained from one of the parents.
She was diagnosed as having MEN1 syndrome at the age of 11 years, when she showed slowed growth, no pubertal development, and wide incomplete bitemporal hemianopia. Magnetic resonance imaging (MRI) of the pituitary gland revealed a pituitary macroadenoma with horizontal, anteroposterior, and vertical diameters of 20, 19 and $24 \mathrm{~mm}$, respectively. The tumor compressed and displaced the optic chiasm, but there were no signs of any extension into the cavernous sinuses.

Laboratory tests showed marked hyperprolactinemia (1800 ng/mL; normal values, 5-25 ng/mL), hypercalcemia, growth hormone $(\mathrm{GH})$ deficiency, and a low level of insulin-like growth factor I (IGF-I). Treatment was started with the longacting dopamine agonist cabergoline, the dose of which was gradually increased to $3 \mathrm{mg} /$ week. Prolactin (PRL) levels decreased to about $100 \mathrm{ng} / \mathrm{mL}$ and the visual field defect normalized, but a repeat MRI showed only marginal shrinkage of the top part of the tumor.

Thyroid and parathyroid ultrasonography revealed an apparently homogeneous thyroid parenchyma, but also a $13 \times 7-\mathrm{mm}$ oval mass at the base of the right thyroid lobe that was suspected of being a parathyroid lesion. The results of adrenal and pancreas computed tomography (CT) were negative.

Thyroid scintigraphy Technetium-99m-methoxyisobutylisonitrile (MIBI) showed accumulation within the lower pole of the right thyroid lobe, thus confirming the presence of a parathyroid adenoma.

It was impossible to make a cytologic diagnosis on the basis of the findings of thyroid fine needle aspiration because the cell sample was inadequate, but high parathyroid hormone $(\mathrm{PTH})$ levels $(>2,000 \mathrm{ng} / \mathrm{mL})$ were found in the liquid. Given the provisional diagnosis of primary hyperparathyroidism, the patient was clinically diagnosed as having MEN1 syndrome, although mutation screening of MEN1 exons 2-10 by means of denaturing gradient gel electrophoresis and direct sequencing was negative.

Total parathyroidectomy with the autografting of a small piece of parathyroid gland into the left forearm was performed at another hospital. The pathologic diagnosis was parathyroid hyperplasia.

Recombinant $\mathrm{GH}$ replacement therapy was considered because the persistent GH deficit led to stunted growth, but it was not started because of the presence of the pituitary tumor, which was subsequently removed by means of transsphenoidal surgery. After surgery, continuous treatment with cabergoline normalized the patient's PRL levels $(20 \mathrm{ng} / \mathrm{mL})$ and, as MRI showed that the pituitary tumor had been almost completely excised, GH replacement therapy was started.

During the next 2 years, PRL levels gradually increased to $65 \mathrm{ng} / \mathrm{mL}$, and MRI showed that the pituitary mass was slowly re-growing despite the continuation of the cabergoline therapy. With the agreement of the patient and her parents, no radiation therapy was administered because of the fear of side effects, but the tumor was closely monitored with the aim of reserving the option of a second surgical procedure in the case of compressive symptoms or excessive tumor growth.

Three years later, when the patient was 14 years old, ultrasonography revealed a subcutaneous $3 \times 3.1-\mathrm{cm}$ lipoma on the right thigh. This soon became larger, and another lipoma was found on the front wall of the left hemithorax. After surgical excision, the first lipoma was histologically 
classified as a spindle cell lipoma (a tumor characterized by the proliferation of collagen-forming spindle cells).

By the time the patient was 15 years old, spontaneous puberty had still not occurred because of the persisting hyperprolactinemia (PRL levels were about $100 \mathrm{ng} / \mathrm{mL}$ ) or the destruction of the normal pituitary gland. As another thorough examination (including a total body CT scan and duodeno-pancreatic echo-endoscopy) did not reveal the presence of a neuroendocrine tumor, the patient was primed with estrogens to induce pubertal changes and the development of secondary sexual characteristics. At the end of the priming period, oral estrogens/progestins were started to treat secondary hypogonadism, and $\mathrm{GH}$ replacement therapy was discontinued.

During the next 4 years, the patient experienced the recurrence of mild hypercalcemia with borderline high $\mathrm{PTH}$ levels, her PRL levels gradually increased to $180 \mathrm{ng} / \mathrm{mL}$, and the vertical diameter of the pituitary tumor increased to $18 \mathrm{~mm}$. Another trans-sphenoidal surgical procedure was performed for the purpose of partial debulking, but a small portion of residual tumor remaining in contact with the optic pathway precluded the use of gamma knife radiosurgery. The patient refused fractionated radiotherapy, and she continued taking cabergoline at a dose of $3 \mathrm{mg} /$ week.

When the patient was 22 years old, abdominal CT revealed the presence of small hypodense pancreatic lesions, suggesting a pancreatic neuroendocrine tumor. Cytologic examination of a specimen taken from the largest lesion under echo-endoscopic guidance (maximum diameter, $\sim 1 \mathrm{~cm}$ ) confirmed the existence of a non-functioning neuroendocrine tumor, and the patient was therefore treated subcutaneously (s.c.) with Long-Action Octreotide Formulation (octreotide LAR) 30 mg every 28 days.

At the time of the last follow-up examination, 1 year after starting octreotide LAR, there was no change in the size of the pancreatic masses, and no new lesions had appeared; the pituitary tumor was slowly re-growing at the same apparent speed as in previous years.

\section{Conventional cytogenetics}

The patient's peripheral blood lymphocytes and the cells of the first lipoma were cytogenetically analyzed using standard methods. A post-surgical sample of fresh lipoma tissue was immediately processed by means of direct and short-term cultures after treatment with pronase-E $(1 \mathrm{mg} / \mathrm{mL}$ at room temperature for 15 minutes). The chromosomes were studied using the QFQ banding technique, and the karyotype was described in accordance with the ISCN (2009) (24).

\section{Fluorescence in situ hybridization (FISH)}

FISH for chromosomes 11 and 3 was performed on metaphases using whole-chromosome painting (WCP) according to the manufacturer's protocols (Abbott Molecular, Des Plains, IL). To characterize the 11q breakpoint, two contiguous BAC clones (RP11-885P1 and RP11-548G17) located in band 11q13.1 were selected using the UCSC Genome Browser (http://www.genome.ucsc.edu/, released March 2006) and provided by Prof. M. Rocchi (University of Bari, Italy). The BAC FISH experiments were performed using the protocols of Lichter et al. (25) and Lichter and Cremer (26), with minor modifications.

\section{aCGH}

Genomic DNA was extracted from the proband's blood and adenoma tissue using the DNeasy Blood \& Tissue Kit (Qiagen, Hilden, Germany), as instructed by the manufacturer. Pooled DNA from the peripheral blood of 10 gendermatched healthy donors (Promega, Madison, WI) was used as a reference for the patient's peripheral blood and adenoma tissue DNA, and the DNA extracted from the patient's peripheral blood was then used as a second reference for the DNA extracted from the adenoma tissue.

Genomic DNA (3 $\mu \mathrm{g}$ ) was hybridized to the $244 \mathrm{~K}$ microarray (Agilent Technologies, Palo Alto, CA), which consisted of approximately 236,000 60-mer oligonucleotide probes covering the entire genome at an average spatial resolution of about 30 kilobases $(\mathrm{kb})$, and was processed according to the manufacturer's instructions. Dye emission was captured by a dual-laser Agilent scanner, and the images were processed using Agilent Feature Extraction software 9.1, and analyzed with DNA Analytics 4.0 software. $A \log _{2}$ ratio plot between the test and reference genomic DNA was assigned in such a way that any aberrations in the test DNA copy number at a particular locus were indicated by the deviation of the plots from a modal value of 0 , and the calls were identified using the ADM-2 algorithm.

The text aberration summaries were analyzed to calculate the medium $\log _{2}$ ratios of every copy number alteration, and distinguish mosaic and non-mosaic alterations. The expected $\log _{2}$ ratio cut-off values for non-mosaic heterozygous and homozygous deletions are -1 and -4 , respectively. The expected $\log _{2}$ ratio cut-off values for non-mosaic duplications and triplications are +0.5 and +1 , respectively. Loweraverage $\log _{2}$ ratios identified cell mosaics: e.g., a $\log _{2}$ ratio of -0.3 represented a cell mosaic of about $50 \%(50 \%$ anomalous and normal cells), as demonstrated by dilution experiments (not shown).

\section{Results}

\section{Constitutional molecular karyotype of the MEN1 patient}

Array CGH analysis of genomic DNA extracted from the patient's peripheral blood showed a heterozygous deletion on chromosome band $11 \mathrm{q} 13.1$ that spanned at least $22.23 \mathrm{~kb}$ (chr11:64320682-64342914 base pairs), and contained all of the MEN1 gene and the $5^{\prime}$ end of the adjacent $M A P 4 K 2$ gene (Figure 1). The centromeric breakpoint mapped to within a $3.7 \mathrm{~kb}$ region between probes A_14_P135243 (normal) and A_16_P02458082 (deleted) (Chr11:64,316, 924-64,320,682 bp, UCSC, hg18), whereas the distal breakpoint mapped to within a $7.7 \mathrm{~kb}$ region between probes A_16_P02458112 (deleted) and A_16_P19263781 (normal) (Chr11: 64,342,914-64,350,624 bp, UCSC, hg18). Furthermore, the patient also carried three copy number polymorphisms (CNPs): a deletion on $11 \mathrm{q} 11$ (chr11:55134486-55187341) and a duplication on $14 q 11.2$ 


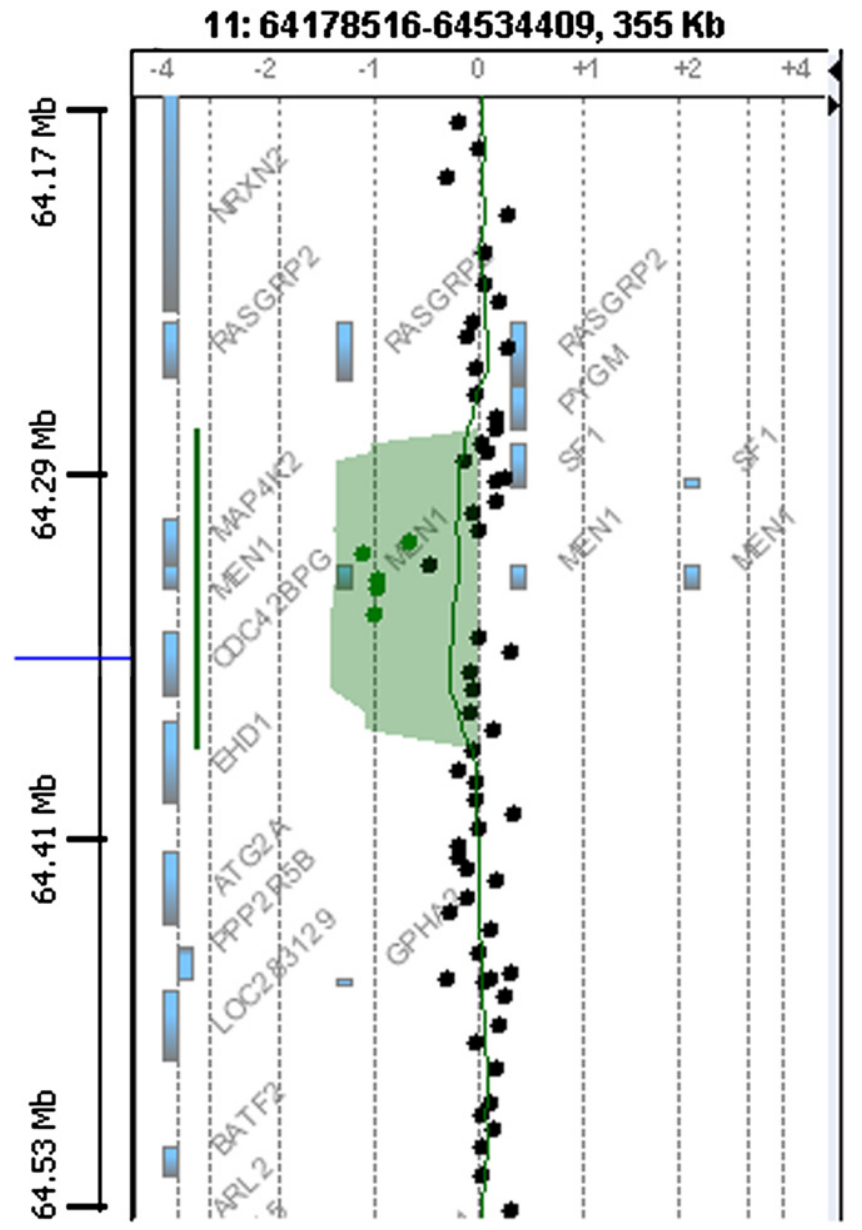

Figure 1 Array CGH detection of a MEN1 gene deletion. The array profile concentrated on a $355 \mathrm{~kb}$ window within the 11q13.1 interval containing the MEN1 deletion, and scatter plot analysis revealed a deletion in 11q13.1 (a horizontal shift to the left of 0 ). Each point represents a single probe. The $\log _{2}$ ratio was plotted for all of the oligonucleotide probes on the basis of their chromosome positions. The figure shows the aberration calls identified using the ADM-2 algorithm (color-shaded areas).

(chr14:19154992-19490689), both of which contained an olfactory receptor cluster, and a duplication on chromosome 15q11.2 (chr15:18683110-20079994) (Supplementary Figure S1).

No parental DNA was available for testing, although we did not expect to find the MEN1 deletion because they were both asymptomatic.

\section{Pituitary adenoma characterization}

The experiments were performed using two different references: the first was pooled gender-matched DNA from healthy controls, and the second was DNA extracted from the patient's peripheral blood to highlight tumor-specific rearrangements without the background of the patient's constitutive copy number variants. As expected, aCGH analysis of the adenoma tissue showed the germline deletion on chromosome band 11q13.1 in both experiments, but the $\log _{2}$ ratio signal of $<-1$ indicated the presence of about
0.5 copies against the two copies present in the reference DNA (median $\log _{2}$ ratio between -2 and -4 ; Figure $2, A$ and $B$ ).

We also found that the CNP on 11q11 had the same MEN1 $\log _{2}$ ratio profile shift to the left of -1 (median $\log _{2}$ ratio between -2 and -4 ; see Supplementary Figures S1 A2 and S1 A3), whereas the shift profile of the two CNPs located on $14 q 11.2$ and $15 q 11.2$ was not different from that of the patient's blood (see Supplementary Figures S1 B2 and S1 C2).

There were also gross copy number aberrations (including the partial loss of 1p), losses of chromosomes 11, 1718 , and 22, as well as gains of chromosomes 1q, 5, 6, 9, 15 , and $X$ (Figure 3 ). In addition, aCGH analysis revealed cytogenetic heterogeneity within the tumor. As shown in Figure 3, each of the chromosomes or chromosomal regions involved in the numerical aberrations showed a specific vertical up or down shift in the $\log _{2}$ ratio profile, which indicated the presence of subclones in quantitatively different ratios. The prevalent chromosomal aberrations were the loss of 1 p12-1pter, the loss of chromosomes 11 and 17, and the gains of chromosomes 6,9 , and 15, all of which indicate a high percentage of affected cells (Supplementary Figure S2).

\section{Lipoma cytogenetics and FISH characterization}

Cytogenetic investigation revealed a 46,XX,t(3;11)(p21;q13) karyotype in all of the analyzed lipoma metaphases (Figure 4A), whereas the patient's constitutional karyotype looked normal.

Specific WCP 11 and WCP 3 paints confirmed the reciprocal translocation $\mathrm{t}(3 ; 11)(\mathrm{p} 21 ; \mathrm{q} 13)$ (Figure $4 \mathrm{~B})$, and $\mathrm{FISH}$ analyses of tumor metaphases using chromosome 11q13.1specific BAC probes made it possible to characterize the $11 \mathrm{q}$ translocation breakpoint. In particular, RP11-885P1 (which contains the MEN1 gene) gave only one signal on the normal chromosome 11, thus revealing the presence of a concomitant deletion at the translocation breakpoint on the der(11) chromosome (Figure 4C). RP11-548G17, which is located contiguously distal to RP11-885P1, allowed the translocation breakpoint to be mapped to 11q13.1, proximally to its target region, as it gave a signal on chromosome $\operatorname{der}(3)$ and the normal chromosome 11 (Figures 4, D and E). After the FISH study, the karyotype could be defined as $46, X X, t(3 ; 11)(p 21 ; q 13)$. ish $\operatorname{der}(3) t(3 ; 11)(p 21 ; q 13.1)(w c p 3+$, wpc11+,RP11-548G17+), $\operatorname{der}(11) t(3 ; 11)(p 21 ; q 13.1)(w p c 3+$, wpc11+,RP11-548G17-,RP11-885P1-).

Using RP11-885P1 on the metaphase spreads of the patient's peripheral blood, FISH gave two identically intense hybridization signals on chromosome 11 because the constitutive approximately $22.23 \mathrm{~kb}$ deletion was too small to be detected by means of BAC FISH.

\section{Discussion}

Array $\mathrm{CGH}$ analysis revealed a germline deletion of the entire MEN1 gene in our patient with sporadic MEN1 syndrome, who was negative for MEN1 mutations upon direct DNA sequencing. The deletion ( $\geq 22.23 \mathrm{~kb}$ ) may be longer at the ends because of the $11 \mathrm{~kb}$ gaps between the flanking oligonucleotides showing a normal hybridization signal and those delimiting the deletion. Interestingly, both of the gap 
A

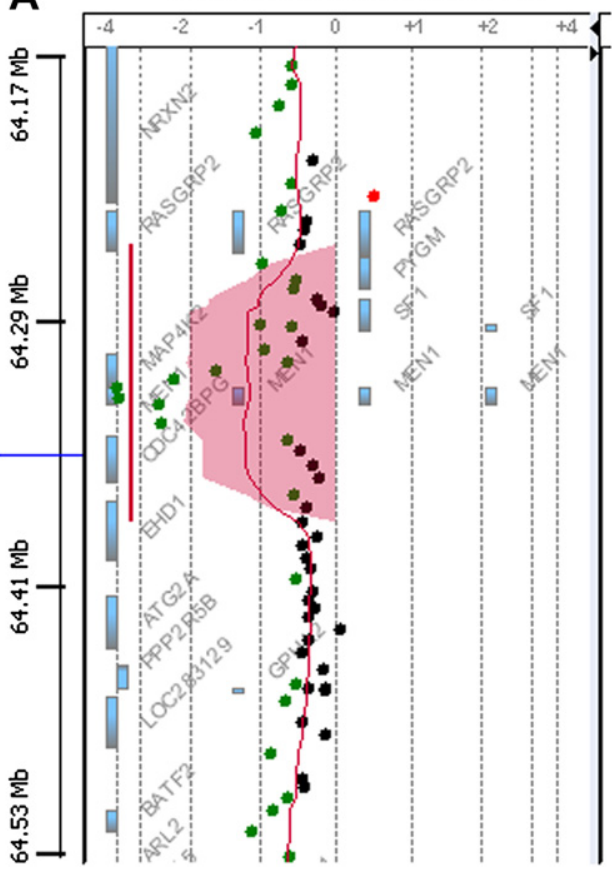

B

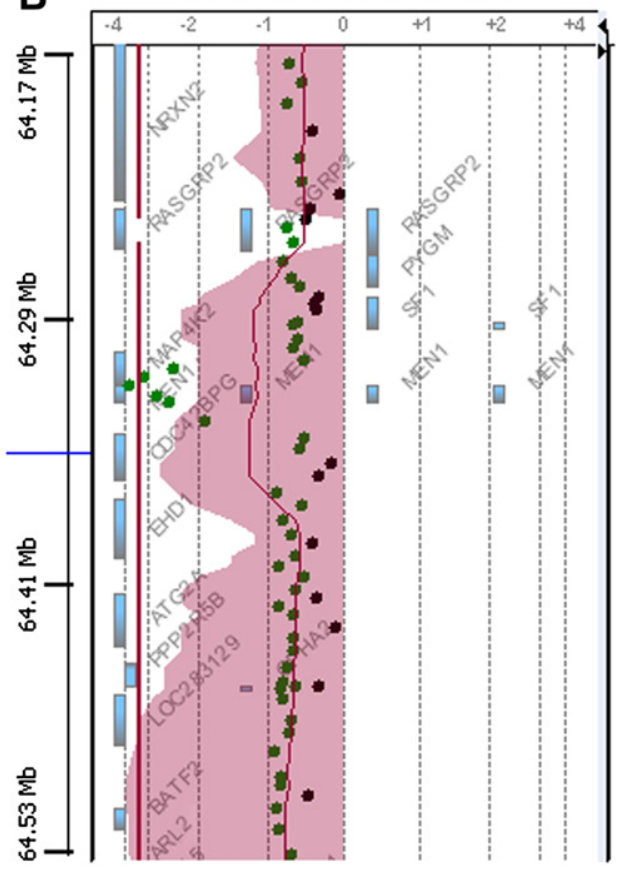

Figure 2 MEN1 array profile of the pituitary adenoma DNA. The array profile concentrated on a $355 \mathrm{~kb} 11 \mathrm{q} 13.1 \mathrm{interval}$ containing the MEN1 gene deletion, and scatter plot analysis revealed a deletion in 11q13.1 with a horizontal shift to the left of -1 (to a log ratio between -2 and -4 ). (A) The 11q13 profile obtained using the patient's peripheral blood DNA as reference. (B) The profile obtained using pooled DNA from the peripheral blood of 10 healthy donors as reference.

regions not covered by the array oligonucleotide probes contain several Alu sequences belonging to the same subfamily that has been described previously as mediating the deletion in MEN1 patients (19).

Array $\mathrm{CGH}$ analysis of the pituitary adenoma revealed the absence of MEN1 alleles in at least some tumor cells because the median $\log _{2}$ ratio of between -2 and -4 indicates the presence of about 0.5 copies, against the two copies in the reference DNA. In addition, a CNP on $11 q 11$ (which involves an olfactory receptor cluster) had the same MEN1 profile shift (see Supplementary Figure 1A), but not the two CNPs located on chromosomes 14 and 15 (see Supplementary Figure 1, B and C). Moreover, in line with previous $\mathrm{CGH}$ findings concerning MEN1 syndrome-related pituitary tumors (27), aCGH showed that the entire chromosome 11 was lost in a large number of cells.

Loss of the wild-type MEN1 allele can be driven by at least three mechanisms: 1) the loss of a whole chromosome 11 as a result of mitotic nondisjunction; 2) the loss of chromosome 11 followed by reduplication; and 3) mitotic recombination proximal to the $11 q 11$ olfactory receptor cluster. The hypothesis that best fits our overall results is the loss of the

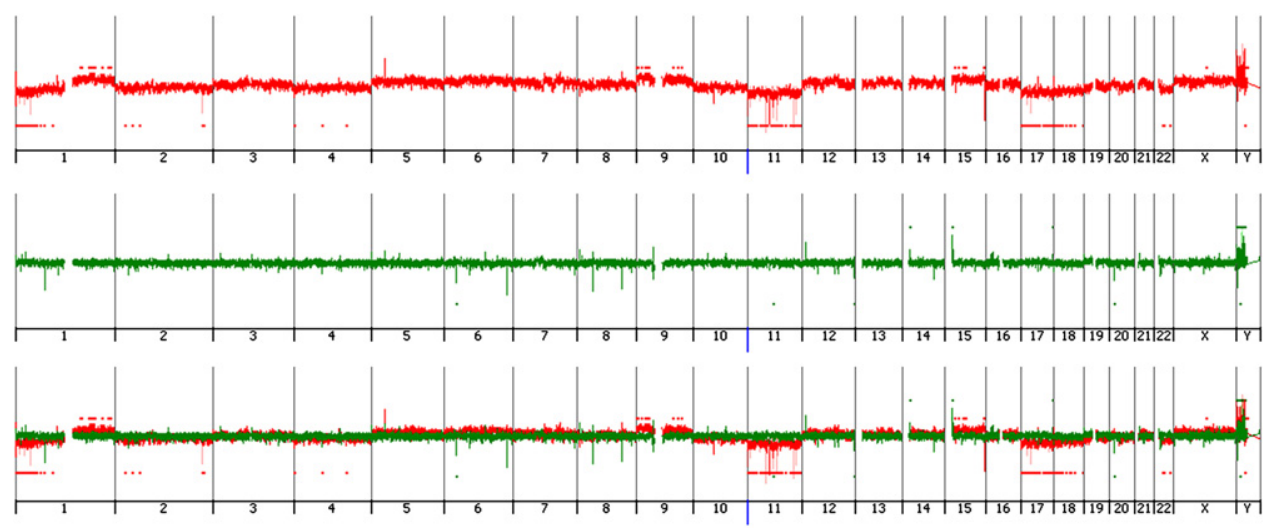

Figure 3 Array CGH profile of the pituitary adenoma. The whole genomic aCGH profile of pituitary adenoma DNA (red) and DNA from the patient's blood (green): the two profiles are superimposed in the bottom panel. The oligonucleotide probes are shown in order from chromosome 1 to 22 within each chromosome. The $\log _{2}$ ratio was plotted for all of the oligonucleotide probes on the basis of their chromosome positions; the vertical bars indicate the separation of the chromosomes. 

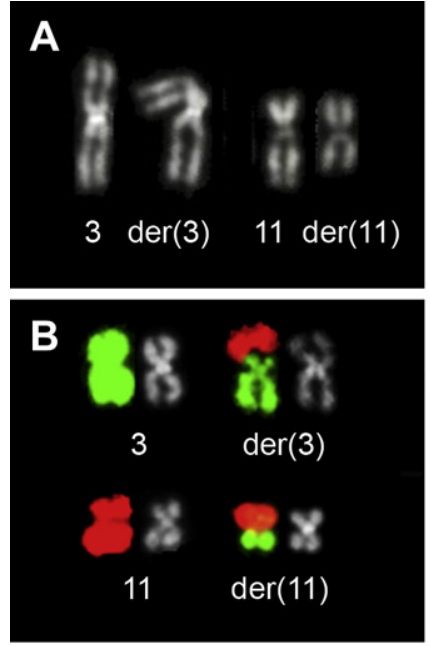

64350000

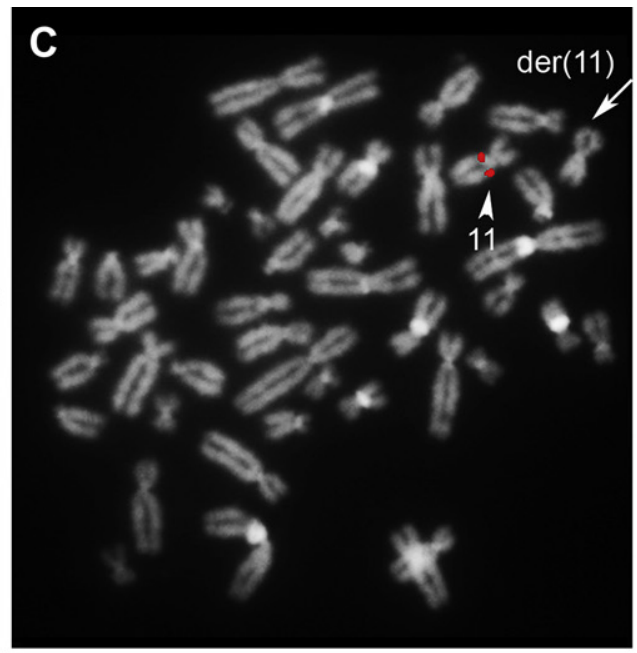

644000001

64450000 I

\begin{abstract}
645000001
\end{abstract}

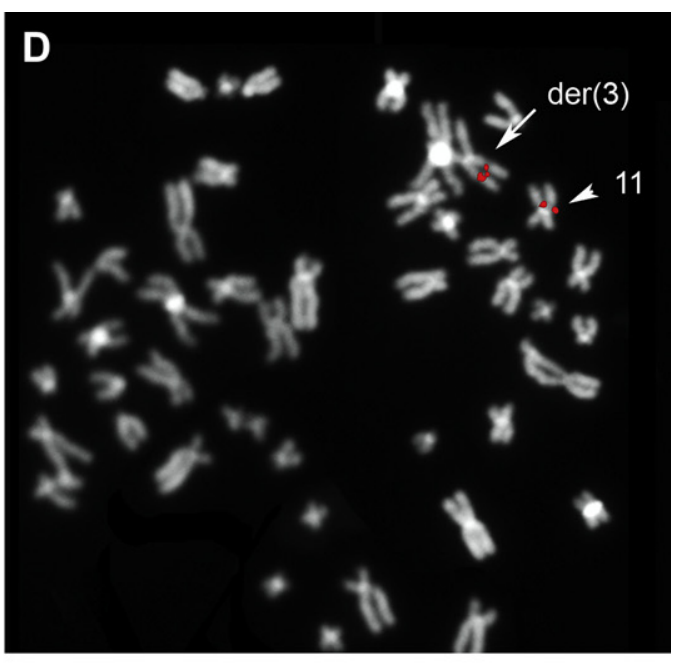

64550000 l

64600000

E

RP11-548G17

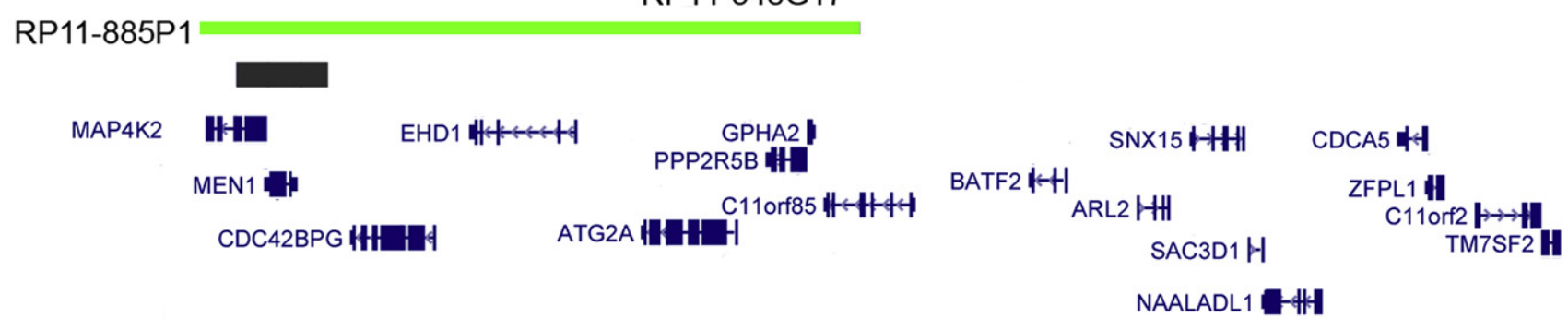

Figure 4 Lipoma cytogenetics and FISH characterization. (A) Partial metaphases showing chromosomes 3 and 11 involved in the translocation. (B) FISH with WCP 3 (green) and WCP 11 (red) on chromosomes 3 and 11. (C) BAC RP11-885P1 (11q13.1) hybridization showing a signal on chromosome 11 (arrow) but not on the der(11) (arrowhead), thus confirming the presence of a MEN1 deletion associated with a targeted translocation event. (D) BAC RP11-548G17 (11q13.1) showing a signal on chromosome 11 (arrowhead) and der(3) (arrow) that targets the boundary of the 11q region translocated onto the chromosome der(3). (E) Physical map of the chromosome 11q13.1 breakpoint of the $t(11 ; 3)(q 13.1 ; p 21)$. The genomic clones used for the FISH experiments are indicated by a green line; the black lines show the region constitutively deleted in the patient; the known UCSC genes are shown in blue. The scale refers to the March 2006 human draft sequence (hg18) from the UCSC genome browser (http://genome.ucsc.edu/ cgi-bin/hgGateway?org=Human).

normal chromosome 11 followed by reduplication of the chromosome bearing the MEN1 deletion.

The cytogenetic heterogeneity in the tumor revealed by aCGH analysis bears witness to clonal evolution, and the additional large copy number aberrations correlate well with previous aCGH and FISH findings (27-32). In particular, the three chromosomal aberrations observed in a large number of cells (the 1p12 1pter and chromosome 11 and 17 losses) coincide with the most frequent cytogenetic changes identified in previous studies $(27,28)$.

The aCGH findings indicate a hyperdiploid karyotype, but do not correlate with the complex karyotype $(56 \sim 79, \mathrm{XX},+\mathrm{X}$ $[2],+1[3],+7[2],+8[3],+9[3],+11[3],+13[2],+14[3],+16[3],+20$ [3],+22[2][cp3]; this suggests a near-triploid set, which has been identified previously by karyotyping, although restricted to only three metaphases (33).

Cytogenetic and FISH studies of the lipoma identified a $t(3 ; 11)(p 21 ; q 13)$ and a concomitant deletion involving the MEN1 gene at the translocation breakpoint on der(11). BAC FISH did not reveal the small constitutive MEN1 deletion, but we hypothesize that the chromosome 11 involved in the translocation was the one carrying the wild-type MEN1 allele.

We could not characterize the 3p21 translocation breakpoint by means of BAC FISH because of the lack of metaphases. The cytogenetic data so far collected in the Mitelman Database of Chromosome Aberrations in Cancer (http:// www.cgap.nci.nih.gov/Chromosomes/Mitelman) show that chromosome deletions on $3 p$ are frequent in the majority of solid tumors and many lymphoid malignancies. Moreover, a number of studies have demonstrated that the 3p21 band is highly prevalent in breakage-prone tumors (34), probably because of its intrinsic fragility (35).

In conclusion, detecting gross deletions in the MEN1 gene in apparently mutation-negative patients who are clinically suspected of being affected by MEN1 is important because of their potential diagnostic value and the contributions they can make to clinical management.

The results of our combined cytogenetic, FISH, and aCGH studies of the adenoma and lipoma tissues are consistent with the MEN1 recessive oncogenesis model, and 
highlight the multiple routes underlying the two hits of MEN1 oncogenesis.

\section{Acknowledgments}

The authors would like to thank the patient's family for their collaboration, as well as Prof. M. Rocchi for providing the RPC11 BACs. This study was supported by a Ministry of Health grant "Ricerca Corrente" to Istituto Auxologico Italiano IRCCS (08C601).

\section{Supplementary data}

Supplementary data associated with this article can be found in the online version at 10.1016/j.cancergen.2011.03.006.

\section{References}

1. Balogh K, Patócs A, Majnik J, et al. Unusual presentation of multiple endocrine neoplasia type 1 in a young woman with a novel mutation of the MEN1 gene. J Human Genet 2004;49: 380-386.

2. Marini F, Falchetti $A$, Del Monte $F$, et al. Multiple endocrine neoplasia type 1. Orphanet J Rare Dis 2006;2:1-38.

3. Peppa M, Boutati E, Kamakari S, et al. Novel germline mutations of the MEN1 gene in Greek families with multiple endocrine neoplasia type 1. Clin Endocrinol 2008;70:75-81.

4. Hai N, Aoki N, Shimatsu A, et al. Clinical features of multiple endocrine neoplasia type 1 (MEN1) phenocopy without germline MEN1 gene mutations: analysis of 20 Japanese sporadic cases with MEN1. Clin Endocrinol 2000;52:509-518.

5. Kouvaraki MA, Lee JE, Shapiro SE, et al. Genotype-phenotype analysis in multiple endocrine neoplasia type 1. Arch Surgery 2002;137:641-647.

6. Chakrabarti R, Srivatsan ES, Wood TF, et al. Deletion mapping of endocrine tumors localizes a second tumor suppressor gene on chromosome band 11q13. Genes Chromosomes Cancer 1998;22:130-137.

7. Cavaco BM, Domingues R, Bacelar MC, et al. Mutational analysis of Portuguese families with multiple endocrine neoplasia type 1 reveals large germline deletions. Clin Endocrinol 2002;56: 465-473.

8. Brandi ML, Gagel RF, Angeli A, et al. Guidelines for diagnosis and therapy of MEN type 1 and type 2. J Clin Endocrinol Metabol 2001;86:5658-5671.

9. Agarwal SK, Lee Burns A, Sukhodolets KE, et al. Molecular pathology of the MEN1 gene. Ann NY Acad Sci 2004;1014: 189-198.

10. Vidal A, Iglesias MJ, Fernández $B$, et al. Cutaneous lesions associated to multiple endocrine neoplasia syndrome type 1 . J Eur Acad Dermatol Venereol 2008;22:835-838.

11. Chandrasekharappa SC, Guru SC, Manickam P, et al. Positional cloning of the gene for multiple endocrine neoplasia-type 1. Science 1997;276:404-407.

12. Agarwal SK, Kennedy PA, Scacheri PC, et al. Menin molecular interactions: insights into normal functions and tumorigenesis. Hormone Metabol Res 2005;37:369-374.

13. Balogh K, Rácz K, Patócs A, Hunyady L. Menin and its interacting proteins: elucidation of menin function. Trends Endocrinol Metabol 2006;17:357-364. review.

14. Lemos MC, Thakker RV. Multiple endocrine neoplasia type 1 (MEN1): analysis of 1336 mutations reported in the first decade following identification of the gene. Human Mutation 2008;29: 22-32.
15. Yang $Y$, Hua $X$. In search of tumor suppressing functions of menin. Mol Cell Endocrinol 2007;265/266:34-41.

16. Pannett AA, Thakker RV. Somatic mutations in MEN type 1 tumors, consistent with the Knudson "two-hit" hypothesis. J Clin Endocrinol Metabol 2001;86:4371-4374.

17. Stenson PD, Ball EV, Mort M, et al. Human Gene Mutation Database (HGMD): 2003 update. Human Mutat 2003;21: 577-581.

18. Owens M, Ellard S, Vaidya B. Analysis of gross deletions in the MEN1 gene in patients with multiple endocrine neoplasia type 1. Clin Endocrinol 2008;68:350-354.

19. Fukuuchi A, Nagamura $Y$, Yaguchi $H$, et al. A whole MEN1 gene deletion flanked by Alu repeats in a family with multiple endocrine neoplasia type 1. J Japan Clin Oncol 2006;36:739-744.

20. Kikuchi M, Ohkura N, Yamaguchi K, et al. Gene dose mapping delineated boundaries of a large germline deletion responsible for multiple endocrine neoplasia type 1. Cancer Lett 2004;208: 81-88.

21. Ellard S, Hattersley AT, Brewer CM, Vaidya B. Detection of an MEN1 gene mutation depends on clinical features and supports current referral criteria for diagnostic molecular genetic testing. Clin Endocrinol 2005;62:169-175.

22. Cardinal JW, Bergman L, Hayward N, et al. A report of a national mutation testing service for the MEN1 gene: clinical presentations and implications for mutation testing. J Med Genet 2005; 42:69-74.

23. Klein RD, Salih S, Bessoni J, Bale AE. Clinical testing for multiple endocrine neoplasia type 1 in a DNA diagnostic laboratory. Genetics in Medicine: official journal of the American College of Medical Genetics 2005;7:131-138.

24. Shaffer LG, Slovak ML, Campbell LJ, editors. ISCN: An international System for Human Cytogenetic Nomenclature. Basel: S. Karger; 2009.

25. Lichter P, Tang CJ, Call K, et al. High-resolution mapping of human chromosome 11 by in situ hybridization with cosmid clones. Science 1990;247:64-69.

26. Lichter $\mathrm{P}$, Cremer T. Chromosome analysis by non-isotopic in situ hybridization. In: Rooney E, Czipolkowski BH, editors. Human Cytogenetics - A Practical Approach, Oxford. University Press; 1992. pp. 157-192.

27. Trautmann K, Thakker RV, Ellison DW, Ibrahim A, Lees PD, Harding $\mathrm{B}$, et al. Chromosomal aberrations in sporadic pituitary tumors. Int J Cancer 2001;91:809-814.

28. Pack SD, Qin LX, Pak E, et al. Common genetic changes in hereditary and sporadic pituitary adenomas detected by comparative genomic hybridization. Genes Chromosomes Cancer 2005;43:72-82.

29. Daniely M, Aviram A, Adams EF, Buchfelder M, Barkai G, Fahlbusch $\mathrm{R}$, et al. Comparative genomic hybridization analysis of nonfunctioning pituitary tumors. J Clin Endocrinol Metabol 1998;83:1801-1805.

30. Harada K, Nishizaki T, Ozaki S, et al. Cytogenetic alterations in pituitary adenomas detected by comparative genomic hybridization. Cancer Genet Cytogenet 1999;112:38-41.

31. Finelli $P$, Giardino $D$, Rizzi N, et al. Non-random trisomies of chromosomes 5,8 and 12 in the prolactinoma sub-type of pituitary adenomas: conventional cytogenetics and interphase FISH study. Int J Cancer 2000;86:344-350.

32. Szymas J, Schluens K, Liebert W, Petersen I. Genomic instability in pituitary adenomas. Pituitary 2002;5:211-219.

33. Finelli $P$, Pierantoni GM, Giardino D, et al. The high mobility group A2 gene is amplified and overexpressed in human prolactinomas. Cancer Res 2002;62:2398-2405.

34. Kost-Alimova M, Imreh S. Modeling non-random deletions in cancer. Sem Cancer Biol 2007;17:19-30.

35. Durkin SG. Glover TW.Chromosome fragile sites. Annu Rev Genet 2007;41:169-192. review. 